\title{
Quantum Riemann Surfaces I. The Unit Disc*
}

\author{
Slawomir Klimek and Andrzej Lesniewski \\ Harvard University, Cambridge, MA 02138, USA \\ Received April 10, 1991; in revised form September 20, 1991
}

\begin{abstract}
We construct a non-commutative $\mathbb{C}^{*}$-algebra $C_{\mu}(\bar{U})$ which is a quantum deformation of the algebra of continuous functions on the closed unit disc $\bar{U} . C_{\mu}(\bar{U})$ is generated by the Toeplitz operators on a suitable Hilbert space of holomorphic functions on $U$.
\end{abstract}

\section{Introduction}

Alain Connes has shown [8] that a substantial part of differential geometry can be extended to a non-commutative setup in which a non-commutative $*$-algebra replaces an algebra of functions on a manifold. Clearly, not every non-commutative algebra has an interesting geometry and, while a satisfactory concept of a "noncommutative differentiable manifold" has not been formulated yet, it is desirable to study examples of such structures.

Recently, a growing number of examples of "non-commutative differentiable manifolds" has been studied, see e.g. $[16,18,22]$. These examples form, in a sense, a testing ground for probing general concepts of non-commutative differential geometry and so give us a better insight into the properties of a non-commutative manifold. Also, some important applications of these ideas have been found in other areas of mathematics and physics $[3,7,9]$.

In this paper, we begin a program of developing a theory of non-commutative Riemann surfaces. We believe that non-commutative Riemann surfaces should play a distinguished role in non-commutative differential geometry, very much like ordinary Riemann surfaces in the commutative case. Also, there are some speculations [13] that quantum Riemann surfaces might be helpful in studying certain integrable systems arising in string theory.

Conceptually, the simplest method of constructing non-commutative manifolds is the framework of deformation quantization, see e.g. $[2,4,19,22]$. This is the starting point of our approach.

* Supported in part by the National Science Foundation under grant DMS/PHY 88-16214 
Let $M$ be a Riemann surface and let $\omega$ be a symplectic form on $M$. Then, the *-algebra $C^{\infty}(M)$ of smooth functions on $M$ comes equipped with a Poisson bracket:

$$
\left\{f_{2} g\right\}:=\omega^{-1}(d f, d g), \quad f, g \in C^{\infty}(M) .
$$

We wish to construct a family of $\mathbb{C}^{*}$-algebras $\mathscr{A}_{\mu}, 0<\mu<1$, called quantum Riemann surfaces, together with "quantization maps" $T^{(\mu)}: C^{\infty}(M) \rightarrow \mathscr{A}_{\mu}$ such that: (i) $T^{(\mu)}$ is linear and *-preserving and (ii) $\lim _{\mu \rightarrow 0}\left\|\frac{1}{i \mu}\left[T_{f}^{(\mu)}, T_{g}^{(\mu)}\right]-T_{\{f, g\}}\right\|{ }^{(\mu)}=0$. Furthermore, we require that the biholomorphisms of $M$ act on $\mathscr{A}_{\mu}$ and that $\mathscr{A}_{\mu}$ is, roughly speaking, the fixed point algebra of the quantum universal covering of $M$.

In this paper we construct a quantum deformation of the unit disc ( $\equiv$ universal covering space of hyperbolic Riemann surfaces). Our construction draws some ideas from Berezin's paper [4] on quantization by covariant symbols. In a subsequent paper [11] we will describe quantum deformations of hyperbolic Riemann surfaces. In another development [12] we study a two-parameter deformation of the unit disc which is closely related to the quantum group $S U_{q}(1,1)$. We should also mention that quantum Riemann surfaces of genus 0 and 1 have already been studied in the literature, see $[16,20]$ (quantum sphere) and $[8,18]$ (quantum torus). Also, quantizations of the unit disk using various symbolic calculi have been studied (see e.g. $[14,21]$ and references therein).

Recall that the group of biholomorphisms $S U(1,1) / \mathbb{Z}_{2}$ of $U=\{z \in \mathbb{C}:|z|<1\}$ consists of fractional transformations

$$
z \rightarrow(a z+b)(\bar{b} z+\bar{a})^{-1}, \quad|a|^{2}-|b|^{2}=1 .
$$

The (essentially unique) $S U(1,1) / \mathbb{Z}_{2}$-invariant symplectic form on $U$ is

$$
\omega=\frac{i}{2}\left(1-|z|^{2}\right)^{-2} d z \wedge d \bar{z}
$$

with the corresponding Poisson bracket

$$
\{f, g\}=i\left(1-|z|^{2}\right)^{2}(\partial f \bar{\partial} g-\bar{\partial} f \partial g) .
$$

In Sect. II we give the definition of the quantum unit disc $C_{\mu}(\bar{U})$ in terms of generators and construct an $S U(1,1) / \mathbb{Z}_{2}$ action on $C_{\mu}(\bar{U})$. In Sects. III and IV we study the representation theory of $C_{\mu}(\bar{U})$ in terms of the algebra of Toeplitz operators on a suitable Hilbert space. This leads us to structural theorems for the quantum unit disc which are closely related to the Brown-Douglas-Fillmore theory [5]. In Sect. $V$ we show that the $S U(1,1) / \mathbb{Z}_{2}$ action can be implemented on this space by a unitary projective representation of $S U(1,1) / \mathbb{Z}_{2}$. Finally, in Sect. VI we study asymptotic expansions of products of Toeplitz operators and prove that, indeed, $T^{(\mu)}$ satisfies requirement (ii) formulated above.

\section{Quantum Unit Disc}

For $0<\mu<1$ we let $P_{\mu}(\bar{U})$ denote the unital $\mathbb{C}$-algebra generated by two elements $z$ and $\bar{z}$ with the following relation:

$$
[z, \bar{z}]=\mu(I-z \bar{z})(I-\bar{z} z) .
$$


We call $P_{\mu}(\bar{U})$ the algebra of polynomials on the closed quantum unit disc. The goal of this section is to study some general properties of representations of $P_{\mu}(\bar{U})$. We show that $P_{\mu}(\bar{U})$ has a nontrivial universal enveloping $\mathbb{C}^{*}$-algebra. We call this $\mathbb{C}^{*}$-algebra the algebra of continuous functions on the closed quantum unit disc.

Let $\mathscr{H}$ be a Hilbert space and let $\pi: P_{\mu}(\bar{U}) \rightarrow \mathscr{L}(\mathscr{H})$ be a representation of $P_{\mu}(\bar{U})$ by bounded linear operators on $\mathscr{H}$ satisfying the condition $\pi(z)^{*}=\pi(\bar{z})$. By abuse of terminology, we will refer to such representations as $*$-representations (strictly speaking, $P_{\mu}(\bar{U})$ is not a $*$-algebra). Setting $\pi(z)=e^{i \theta}, \pi(\bar{z})=e^{-i \theta}, 0 \leqq \theta<2 \pi$, we see that nontrivial $*$-representations of $P_{\mu}(\bar{U})$ exist. By $\pi\left(P_{\mu}(\bar{U})\right)^{-}$we denote the $\mathbb{C}^{*}$-algebra of operators on $\mathscr{H}$ defined as the norm closure of $\pi\left(P_{\mu}(\bar{U})\right)$.

We will frequently use the following notation:

$$
x:=z \bar{z}, \quad y:=\bar{z} z .
$$

Applying $\pi$ and taking the adjoint of (II.1) we find that

$$
[\pi(x), \pi(y)]=0,
$$

i.e., $\pi(x)$ and $\pi(y)$ generate an abelian subalgebra of $\pi\left(P_{\mu}(\bar{U})\right)$.

Theorem II.1. The operators $\pi(z)$ and $\pi(\bar{z})$ satisfy

$$
\|\pi(z)\|=\|\pi(\bar{z})\|=1 \text {. }
$$

Proof. Let $\mathbb{C}^{*}(x, y)$ denote the abelian unital sub- $\mathbb{C}^{*}$-algebra of $\mathscr{L}(\mathscr{H})$ generated by $\pi(x)$ and $\pi(y)$. As a consequence of a Gelfand-Naimark theorem, $\mathbb{C}^{*}(x, y)$ is isometrically isomorphic with $C(\Sigma)$, the $\mathbb{C}^{*}$-algebra of continuous functions on the spectrum $\Sigma$ of $\mathbb{C}^{*}(x, y)$. Let $x(\sigma)$ and $y(\sigma), \sigma \in \Sigma$, denote the images of $\pi(x)$ and $\pi(y)$ under this isomorphism. Clearly, $x(\sigma), y(\sigma) \geqq 0$.

From Eq. (II.1) we have

$$
y(\sigma)[\mu x(\sigma)+1-\mu]=(\mu+1) x(\sigma)-\mu .
$$

Since $\mu x(\sigma)+1-\mu>0$, we have for all $\sigma \in \Sigma$,

$$
y(\sigma)=\frac{(\mu+1) x(\sigma)-\mu}{\mu x(\sigma)+1-\mu} .
$$

The function $0 \leqq t \rightarrow[(\mu+1) t-\mu] /[\mu t+1-\mu]$ is continuous and monotonically increasing, and thus as a consequence of (II.6),

$$
\|\pi(y)\|=\frac{(\mu+1)\|\pi(x)\|-\mu}{\mu\|\pi(x)\|+1-\mu} .
$$

But from (II.2), $\|\pi(x)\|=\|\pi(y)\|=\|\pi(z)\|^{2}$. Substituting this into (II.7) and solving the resulting algebraic equation yields $\|\pi(z)\|=1$, as claimed.

\section{Lemma II.2.}

$$
\begin{aligned}
& \operatorname{Ker}(I-\pi(x))=\operatorname{Ker}(I-\pi(y)), \\
& \operatorname{Ran}(I-\pi(x))=\operatorname{Ran}(I-\pi(y)) .
\end{aligned}
$$

Proof. (i) This is an immediate consequence of (II.1) and (II.3). 
(ii) If $\psi=(I-\pi(x)) \varphi$, then from (II.1) and (II.3), $(I+\mu(I-\pi(y))) \psi=(I-\pi(y)) \varphi$. But $\|\mu(I-\pi(y))\|<1$, and thus $\psi=(I-\pi(y))(I+\pi(y)))^{-1} \varphi \in \operatorname{Ran}(I-\pi(y))$, proving that $\operatorname{Ran}(I-\pi(x)) \subset \operatorname{Ran}(I-\pi(y))$. By a similar argument, $\operatorname{Ran}(I-\pi(y)) \subset$ $\operatorname{Ran}(I-\pi(x))$.

\section{Lemma II.3.}

(i) $\operatorname{Ker}(I-\pi(x))$ is invariant under $\pi\left(P_{\mu}(\bar{U})\right)^{-}$,

(ii) $\operatorname{Ran}(I-\pi(x))$ is invariant under $\pi\left(P_{\mu}(\bar{U})\right)$.

Proof. (i) Indeed, if $\psi \in \operatorname{Ker}(I-\pi(x))$, then by (II.8), $(I-\pi(x)) \pi(z) \psi=\pi(z)(I-\pi(y)) \psi=$ 0 , and $(I-\pi(y)) \pi(\bar{z}) \psi=\pi(\bar{z})(I-\pi(x)) \psi=0$, i.e., $\pi(z) \psi, \pi(\bar{z}) \psi \in \operatorname{Ker}(I-\pi(x))$. Since $\operatorname{Ker}(I-\pi(x))$ is closed, the claim follows.

(ii) If $\psi=(I-\pi(x)) \varphi \in \operatorname{Ran}(I-\pi(x))$, then by (II.9), $\pi(\bar{z}) \psi=(I-\pi(y)) \pi(\bar{z}) \varphi \in$ $\operatorname{Ran}(I-\pi(y))=\operatorname{Ran}(I-\pi(x))$. Furthermore, $\psi=(I-\pi(y)) \varphi^{\prime}$ and thus $\pi(z) \psi=$ $(I-\pi(x)) \pi(z) \varphi^{\prime} \in \operatorname{Ran}(I-\pi(x))$.

We now write

$$
\mathscr{H}=\operatorname{Ker}(I-\pi(x)) \oplus \stackrel{\mathscr{H}}{ },
$$

where $\mathscr{\mathscr { H }}$ is the orthogonal complement of $\operatorname{Ker}(I-\pi(x))$. As a consequence of Lemma II.3 (i) and the fact that $\pi\left(P_{\mu}(\bar{U})\right)^{-}$is a *-algebra, both direct summands in (II.10) are invariant under $\pi\left(P_{\mu}(\bar{U})\right)^{-}$.

Let now $\gamma \in S U(1,1)$. We will write the inverse of $\gamma$ as

$$
\gamma^{-1}=\left(\begin{array}{cc}
a & b \\
\bar{b} & \bar{a}
\end{array}\right) \quad a, b \in \mathbb{C},
$$

where

$$
|a|^{2}-|b|^{2}=1 \text {. }
$$

Lemma II.4. If $\gamma \in S U(1,1)$, then $(b \pi(z)+a)^{-1} \in \pi\left(P_{\mu}(\bar{U})\right)^{-}$.

Proof. Consider the series

$$
a^{-1} \sum_{n \geqq 0}(-b / a)^{n} \pi(z)^{n}
$$

As a consequence of (II.12), $|b / a|<1$. Since by Theorem II.1, $\|\pi(z)\|=1$, the series (II.13) converges to an element of $\mathscr{L}(\mathscr{H})$. A routine calculation show that its limit is, in fact, $(b \pi(z)+a)^{-1}$.

We now set for $\gamma \in S U(1,1)$,

$$
\begin{aligned}
& \rho_{\gamma}(\pi(z)):=(\bar{a} \pi(z)+\bar{b})(b \pi(z)+a)^{-1}, \\
& \rho_{\gamma}(\pi(\bar{z})):=(\bar{b} \pi(\bar{z})+\bar{a})^{-1}(a \pi(\bar{z})+b) .
\end{aligned}
$$

In the theorem below we assert that $\rho_{\gamma}$ defines an action of $S U(1,1)$ on $\pi\left(P_{\mu}(\bar{U})\right)^{-}$.

Theorem II.5. Let $\gamma \in S U(1,1)$. Then:

$$
\begin{aligned}
\rho_{\gamma}(\pi(z))^{*} & =\rho_{\gamma}(\pi(\bar{z})), \\
{\left[\rho_{\gamma}(\pi(z)), \rho_{\gamma}(\pi(\bar{z}))\right] } & =\mu\left(I-\rho_{\gamma}(\pi(z)) \rho_{\gamma}(\pi(\bar{z}))\right)\left(I-\rho_{\gamma}(\pi(\bar{z})) \rho_{\gamma}(\pi(z))\right) .
\end{aligned}
$$

Proof. (i) This is clear. 
(ii) We verify (II.16) on $\operatorname{Ker}(I-\pi(x))$ and $\stackrel{\mathscr{H}}{ }$ separately. We set $z^{\prime}:=\rho_{\gamma}(\pi(z))$ and $\bar{z}^{\prime}:=\rho_{\gamma}(\pi(\bar{z}))$. If $\omega \in \operatorname{Ker}(I-\pi(x))$, then a straightforward calculation shows that

$$
z^{\prime} \bar{z}^{\prime} \psi=\bar{z}^{\prime} z^{\prime} \psi=\psi
$$

which proves that (II.16) holds on $\operatorname{Ker}(I-\pi(x))$.

To prove that (II.16) holds on $\mathscr{H}$ we observe that the inverses $(I-\pi(x))^{-1}$ and $(I-\pi(y))^{-1}$ exist as densely defined unbounded operators. Furthermore, as a consequence of Lemma II.3 (ii), $\operatorname{Dom}\left((I-\pi(x))^{-1}\right)=\operatorname{Dom}\left((I-\pi(y))^{-1}\right)=$ $\operatorname{Ran}(I-\pi(x))$.

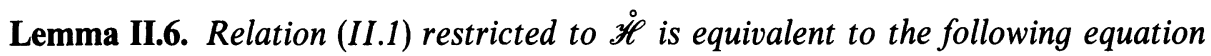
on $\operatorname{Ran}(I-\pi(x))$ :

$$
(I-\pi(x))^{-1}-(I-\pi(y))^{-1}=\mu .
$$

Proof. Multiplying(II.17) by $I-\pi(x)$ on one side and by $I-\pi(y)$ on the other yields (II.1). Conversely, writing (II.1) as

$$
((I-\pi(y))-(I-\pi(x))) \varphi=\mu(I-\pi(x))(I-\pi(y)) \varphi, \quad \varphi \in \stackrel{\mathscr{H}}{ },
$$

and using (II.3) yields (II.17).

We can now conclude the proof of the theorem. As a consequence of (II.12) and Lemma II.4,

$$
I-z^{\prime} \bar{z}^{\prime}=(b \pi(z)+a)^{-1}(I-\pi(x))(\bar{b} \pi(\bar{z})+\bar{a})^{-1},
$$

and

$$
I-\bar{z}^{\prime} z^{\prime}=(\bar{b} \pi(\bar{z})+\bar{a})^{-1}(I-\pi(y))(b \pi(z)+a)^{-1} .
$$

We claim that $I-z^{\prime} \bar{z}^{\prime}$ and $I-\bar{z}^{\prime} z^{\prime}$ are invertible on $\operatorname{Ran}(I-\pi(x))$. Indeed, we set for $\psi \in \operatorname{Ran}(I-\pi(x))$

$$
\left(I-z^{\prime} \bar{z}^{\prime}\right)^{-1} \psi=(\bar{b} \pi(\bar{z})+\bar{a})(I-\pi(x))^{-1}(b \pi(z)+a) \psi,
$$

and

$$
\left(I-\bar{z}^{\prime} z^{\prime}\right)^{-1} \psi=(b \pi(z)+a)(I-\pi(y))^{-1}(\bar{b} \pi(\bar{z})+\bar{a}) \psi .
$$

As a consequence of Lemma II.3 (ii) these are well defined. A simple calculation shows that (II.20) and (II.21) are indeed the inverses of $I-z^{\prime} \bar{z}^{\prime}$ and $I-\bar{z}^{\prime} z^{\prime}$, respectively.

As a consequence of the above considerations we have, as operator equations on $\operatorname{Ran}(I-\pi(x))$ :

$$
\begin{aligned}
\left(I-z^{\prime} \bar{z}^{\prime}\right)^{-1}-\left(I-\bar{z}^{\prime} z^{\prime}\right)^{-1}= & (\bar{b} \pi(\bar{z})+\bar{a})(I-\pi(x))^{-1}(b \pi(z)+a) \\
& -(b \pi(z)+a)(I-\pi(y))^{-1}(\bar{b} \pi(\bar{z})+\bar{a}) \\
= & |b|^{2}\left\{\pi(y)(I-\pi(y))^{-1}-\pi(x)(I-\pi(x))^{-1}\right\} \\
& +|a|^{2}\left\{(I-\pi(x))^{-1}-(I-\pi(y))^{-1}\right\} \\
= & -|b|^{2}\left\{[\pi(z), \pi(\bar{z})](I-\pi(y))^{-1}+\mu \pi(x)\right\}+\mu|a|^{2} \\
= & \mu\left(|a|^{2}-|b|^{2}\right)=\mu .
\end{aligned}
$$

This shows that $z^{\prime}$ and $\bar{z}^{\prime}$ satisfy (II.17) and the proof of the theorem is complete. 
We now let for $a \in P_{\mu}(\bar{U})$,

$$
\|a\|:=\sup _{\pi}\|\pi(a)\|
$$

where the supremum is taken over all possible *-representations of $P_{\mu}(\bar{U})$. As a consequence of Theorem II.1, $\|a\|<\infty$ for all $a \in P_{\mu}(\bar{U})$. Let $N:=\left\{a \in P_{\mu}(\bar{U}):\|a\|=0\right\}$ be the null-ideal in $P_{\mu}(\bar{U})$. We define $C_{\mu}(\bar{U})$ to be the completion of $P_{\mu}(\bar{U}) / N$ in the norm induced by $\|\cdot\|$ and call it the algebra of continuous functions on the closed quantum unit disc. By definition, $C_{\mu}(\bar{U})$ is a unital $\mathbb{C}^{*}$-algebra with involution defined by $z^{*}:=\bar{z}$. The following theorem is a consequence of the above considerations.

Theorem II.7. The $\mathbb{C}^{*}$-algebra $C_{\mu}(\bar{U})$ is generated by two elements $z$ and $\bar{z}$ satisfying (II.1) and such that $\|z\|=1$. The mapping

$$
\gamma \rightarrow \rho_{\gamma}(z):=(\bar{a} z+\bar{b})(b z+a)^{-1}
$$

defines an action of $S U(1,1)$ on $C_{\mu}(\bar{U})$.

\section{Representation Theory}

In this section we classify all irreducible *-representations of the algebra of polynomials on the quantum unit disc $P_{\mu}(\bar{U})$. As in Sect. II, let $\pi: P_{\mu}(\bar{U}) \rightarrow \mathscr{L}(\mathscr{H})$ be a representation of $P_{\mu}(\bar{U})$ on a Hilbert space $\mathscr{H}$.

Proposition III.1. Let $\lambda_{n}:=n \mu(1+n \mu)^{-1}, n=0,1,2, \ldots$ Then

$$
\begin{aligned}
& \operatorname{Spec}(\pi(x)) \subset\left\{\lambda_{n}\right\}_{n \geqq 1} \cup\{1\}, \\
& \operatorname{Spec}(\pi(y)) \subset\left\{\lambda_{n}\right\}_{n \geqq 0} \cup\{1\} .
\end{aligned}
$$

Proof. By Theorem II.1, $\|\pi(x)\|=\|\pi(y)\|=1$. Therefore, $\operatorname{Spec}(\pi(x)), \operatorname{Spec}(\pi(y)) \subset$ $[0,1]$. Furthermore, $\operatorname{Spec}(\pi(x)) \backslash\{0\}=\operatorname{Spec}(\pi(y)) \backslash\{0\}$. Let $I_{n}, n=0,1,2, \ldots$ be a sequence of open subsets of $[0,1]$ defined by $I_{n}:=\left(\lambda_{n}, \lambda_{n+1}\right)$. We claim that $I_{n} \cap \operatorname{Spec}(\pi(x))=\varnothing$ and $I_{n} \cap \operatorname{Spec}(\pi(y))=\varnothing$, for $n \geqq 0$. Indeed, as a consequence of (II.1),

$$
\pi(y)=\{(\mu+1) \pi(x)-\mu\}\{\mu \pi(x)+1-\mu\}^{-1},
$$

and thus $\pi(x) \geqq \lambda_{i}$. Therefore, $I_{0} \cap \operatorname{Spec}(\pi(x))=\varnothing$. Consequently, $I_{0} \cap \operatorname{Spec}(\pi(y))=$ $\varnothing$. We now proceed inductively. Assume that $I_{0}, I_{1}, \ldots, I_{n}$ do not intersect the spectra of $\pi(x)$ and $\pi(y)$. If $\lambda \in I_{n+1}$ is in the spectrum of $\pi(x)$, then by (III.3) $\widetilde{\lambda}=\{(\mu+1) \lambda-\mu\}\{\mu \lambda+1-\mu\}^{-1}$ is in the spectrum of $\pi(y)$. But $\tilde{\lambda} \in I_{n}$, which is a contradiction. Therefore, $I_{n+1}$ does not intersect the spectrum of $\pi(x)$ and, consequently, it does not intersect the spectrum of $\pi(y)$.

Theorem III.2. An irreducible *-representation $(\mathscr{H}, \pi)$ of $P_{\mu}(\bar{U})$ is unitarily equivalent to one of the two following representations:

$(\alpha)$ a one-dimensional representation $\pi(z)=e^{-i \theta}, \pi(\bar{z})=e^{i \theta}$, where $0 \leqq \theta<2 \pi$;

$(\beta)$ an infinite dimensional representation defined as follows: let $\mathscr{H}=\overline{l^{2}}\left(\mathbb{Z}_{+}\right)$(where $\mathbb{Z}_{+}$is the set of nonnegative integers) and let $\left\{f_{n}\right\}_{n \geqq 0}$ be an orthonormal basis for $\mathscr{H}$. 
Then

$$
\begin{aligned}
& \pi(\bar{z}) f_{n}=\left\{\frac{(n+1) \mu}{1+(n+1) \mu}\right\}^{1 / 2} f_{n+1}, \quad n \geqq 0, \\
& \pi(z) f_{n}=\left\{\begin{array}{cl}
0 & n=0, \\
\left\{\frac{n \mu}{1+n \mu}\right\}^{1 / 2} f_{n-1}, & n \geqq 1 .
\end{array}\right.
\end{aligned}
$$

Proof. We assume that $(\mathscr{H}, \pi)$ is an irreducible $*$-representation of $P_{\mu}(\bar{U})$. We write $\mathscr{H}$ as in (II.10) and observe that either $\operatorname{Ker}(I-\pi(x))=0$ or $\stackrel{\mathscr{H}}{=}=0$. If $\dot{\mathscr{H}}=0$, then $\pi(z) \pi(z)^{*}=\pi(z)^{*} \pi(z)=I$, i.e., $\pi(z)$ is unitary. As a consequence, $\mathscr{H}$ is onedimensional and $\pi(z)=e^{-i \theta}$. Let $\operatorname{Ker}(I-\pi(x))=0$ and let $\mathscr{G}_{n}, n=1,2, \ldots$ be the eigenspaces of $\pi(x)$ corresponding to the eigenvalue $\lambda_{n}$. By the spectral theorem, $\mathscr{H}=\bigoplus_{n \geqq 1} \mathscr{G}_{n}$. Observe that, as a consequence of (II.1), $\pi(\bar{z})$ maps $\mathscr{G}_{n}$ into $\mathscr{G}_{n+1}$, and $\pi(z)$ maps $\mathscr{G}_{n+1}$ into $\mathscr{G}_{n}$. Indeed, if $f \in \mathscr{G}_{n}$, then

$$
\begin{aligned}
(I+\mu-\mu \pi(\bar{z} z)) \pi(z \bar{z}) \pi(\bar{z}) f= & \mu \pi(\bar{z}) f-\mu \pi(\bar{z}) \pi(z \bar{z}) f \\
& +\pi(\bar{z}) \pi(z \bar{z}) f=\left(\mu-\mu \lambda_{n}+\lambda_{n}\right) \pi(\bar{z}) f .
\end{aligned}
$$

Consequently,

$$
\begin{aligned}
\pi(z \bar{z}) \pi(\bar{z}) f & =\left(\mu-\mu \lambda_{n}+\lambda_{n}\right)(I+\mu-\mu \pi(\bar{z} z))^{-1} \pi(\bar{z}) f \\
& =\left(\mu-\mu \lambda_{n}+\lambda_{n}\right) \pi(\bar{z})(I+\mu-\mu \pi(z \bar{z}))^{-1} f \\
& =\left(\mu-\mu \lambda_{n}+\lambda_{n}\right)\left(1+\mu-\mu \lambda_{n}\right)^{-1} \pi(\bar{z}) f \\
& =\lambda_{n+1} \pi(\bar{z}) f,
\end{aligned}
$$

i.e., $\pi(\bar{z}) f \in \mathscr{G}_{n+1}$. In the same way we show that $\pi(z): \mathscr{G}_{n+1} \rightarrow \mathscr{G}_{n}$. Now, let $i_{n}$ denote the restriction of $\pi(\bar{z})$ to $\mathscr{G}_{n}$ and let $r_{n}$ denote the restriction of $\pi(z)$ to $\mathscr{G}_{n}$. Then $i_{n} r_{n+1}=\lambda_{n} I$ and $r_{n+1} i_{n}=\lambda_{n} I$. This shows that the subspaces $\mathscr{G}_{n}$ and $\mathscr{G}_{n+1}$ are isomorphic, $n=1,2, \ldots$ We claim that $\mathscr{G}_{1}$ is one-dimensional. Indeed, if $e_{1}, e_{2} \in \mathscr{G}_{1}$ are nonzero and mutually orthogonal, then the subspaces generated by $\left\{\pi\left(\bar{z}^{m}\right) e_{1}\right\}_{m \geqq 1}$ and $\left\{\pi\left(\bar{z}^{m}\right) e_{2}\right\}_{m \geqq 1}$ are nonzero, invariant under $\pi\left(P_{\mu}(\bar{U})\right)$ and mutually orthogonal. As a consequence, all $\mathscr{G}_{n}$ are one-dimensional. Let $f_{0} \in \mathscr{G}_{1}$ with $\left\|f_{0}\right\|=1$, and let $f_{n}, n \geqq 1$, be defined by

$$
f_{n}:=\left\|\pi\left(\bar{z}^{n}\right) f_{0}\right\|^{-1} \pi\left(\bar{z}^{n}\right) f_{0} \in \mathscr{G}_{n+1} .
$$

Then $\left\{f_{n}\right\}_{n \geqq 0}$ forms an orthonormal basis for $\mathscr{H}$. A simple computation shows that

$$
\begin{aligned}
& \pi(\bar{z}) f_{n}=\sqrt{\lambda_{n+1}} f_{n+1}, \quad n \geqq 0, \\
& \pi(z) f_{n}=\left\{\begin{array}{cc}
0 & n=0 \\
\sqrt{\lambda_{n}} f_{n-1}, & n \geqq 1 .
\end{array}\right.
\end{aligned}
$$

We now describe a representation of $P_{\mu}(\bar{U})$ on a Hilbert space of holomorphic functions on the unit disc $U \subset \mathbb{C}$. This space was previously studied by Berezin [4] in the context of quantization of covariant symbols. 
We set

$$
r:=\frac{1}{\mu}+1,
$$

and observe that $r>2$. Let $\mathscr{H}_{U}$ be the Hilbert space of holomorphic functions on $U$ equipped with the Petersson scalar product

$$
(\varphi, \psi):=\frac{i}{2} \int_{U} \varphi(\zeta) \overline{\psi(\zeta)}\left(1-|\zeta|^{2}\right)^{r-2} d \zeta \wedge d \bar{\zeta}
$$

We set

$$
d \mu_{r}(\zeta)=\frac{i}{2}\left(1-|\zeta|^{2}\right)^{r-2} d \zeta \wedge d \bar{\zeta}
$$

It is well known that the functions

$$
\varphi_{n}(\zeta)=\left\{\frac{1}{\pi} \frac{\Gamma(r+n)}{n ! \Gamma(r-1)}\right\}^{1 / 2} \zeta^{n}
$$

form an orthonormal basis for $\mathscr{H}_{U}$. This leads us to the following expression for the corresponding Bergman kernel:

$$
K(\zeta, \eta)=\pi^{-1}(r-1)(1-\zeta \bar{\eta})^{-r} .
$$

Recall that $K(\zeta, \eta)$ is the integral kernel of the orthogonal projection $P$ of $L^{2}\left(U, d \mu_{r}\right)$ onto $\mathscr{H}_{U}$. For each continuous function $f$ on $\bar{U}$ we consider an operator $T_{f}$ on $\mathscr{H}_{U}$ defined by

$$
T_{f} \varphi(\zeta):=\left(P M_{f} \varphi\right)(\zeta)=\int_{U} K(\zeta, \eta) f(\eta) \varphi(\eta) d \mu_{r}(\zeta)
$$

Here $M_{f}$ denotes the multiplication operator on $L^{2}\left(U, d \mu_{r}\right)$. Observe that $T_{f} \in \mathscr{L}\left(\mathscr{H}_{U}\right)$. An operator of the form $T_{f}$ is called a Toeplitz operator with symbol $f$ (see e.g. [10]). Note that $T_{\bar{f}}=T_{f}^{*}$. Let $\mathscr{T}_{\mu}(\bar{U})$ denote the $\mathbb{C}^{*}$-algebra generated by the Toeplitz operators.

We define the following Toeplitz operators:

$$
\begin{aligned}
& (\pi(\bar{z}) \varphi)(\zeta):=\int_{U} K(\zeta, \eta) \eta \varphi(\eta) d \mu_{r}(\eta)=\zeta \varphi(\zeta), \\
& (\pi(z) \varphi)(\zeta):=\int_{U} K(\zeta, \eta) \bar{\eta} \varphi(\eta) d \mu_{r}(\eta)=\left(\pi(\bar{z})^{*} \varphi\right)(\zeta) .
\end{aligned}
$$

Lemma III.3. We have

$$
(\pi(z) \varphi)(\zeta)=\zeta^{-r} \int_{0}^{\zeta} \eta^{r-1} \varphi^{\prime}(\eta) d \eta
$$

Proof. We set $\psi(\zeta):=(\pi(z) \varphi)(\zeta)$. Then using (III.11),

$$
\begin{aligned}
\psi(\zeta) & =\pi^{-1}(r-1) \int_{U} \bar{\eta}(1-\zeta \bar{\eta})^{-r} \varphi(\eta) d \mu_{r}(\eta) \\
& =\pi^{-1} \frac{d}{d \zeta} \int_{U}(1-\zeta \bar{\eta})^{-r+1} \varphi(\eta) d \mu_{r}(\eta)
\end{aligned}
$$




$$
\begin{aligned}
& =\frac{1}{r-1} \frac{d}{d \zeta} \int_{U}(1-\zeta \bar{\eta}) K(\zeta, \eta) \varphi(\eta) d \mu_{r}(\eta) \\
& =\frac{1}{r-1}\left[\varphi^{\prime}(\zeta)-\frac{d}{d \zeta}(\zeta \psi(\zeta))\right]
\end{aligned}
$$

As a consequence, we obtain a differential equation on $\psi$,

$$
\zeta \psi^{\prime}+r \psi=\varphi^{\prime} .
$$

Solving this equation yields

$$
\psi(\zeta)=\zeta^{-r} \int_{0}^{\zeta} \eta^{r-1} \varphi^{\prime}(\eta) d \eta
$$

as claimed.

Theorem III.4. $\left(\mathscr{H}_{U}, \pi\right)$ defines a $*$-representation of $P_{\mu}(\bar{U})$. This representation is unitarily equivalent with representation $(\beta)$ of Theorem III.2.

Proof. From (III.10) and (III.13),

$$
\left(\pi(\bar{z}) \varphi_{n}\right)(\zeta)=\zeta \varphi_{n}(\zeta)=\left\{\frac{n+1}{n+r}\right\}^{1 / 2} \varphi_{n+1}(\zeta)
$$

which proves (III.4). Likewise, from (III.14),

$$
\left(\pi(z) \varphi_{n}\right)(\zeta)=\zeta^{-r} \int_{0}^{\zeta} \eta^{r-1} \varphi_{n}^{\prime}(\eta) d \eta=\left\{\frac{n}{n-1+r}\right\}^{1 / 2} \varphi_{n-1}(\zeta)
$$

for $n \geqq 1$ and $\left(\pi(z) \varphi_{0}\right)(\zeta)=0$. This proves (III.5).

In other words, $\pi$ is a homomorphism of $P_{\mu}(\bar{U})$ into $\mathscr{T}_{\mu}(\bar{U})$. Our next result states that, in fact, $C_{\mu}(\bar{U})$ is isomorphic with ${ }^{\mu} \mathscr{T}_{\mu}(\bar{U})$. Therefore, the concrete $\mathbb{C}^{*}$-algebra $\mathscr{T}_{\mu}(\bar{U})$ may serve as a universal representation of the abstract $\mathbb{C}^{*}$ algebra $C_{\mu}(\bar{U})$.

Theorem III.5. $\pi: P_{\mu}(\bar{U}) \rightarrow \mathscr{T}_{\mu}(\bar{U})$ induces an isomorphism of the $\mathbb{C}^{*}$-algebras $C_{\mu}(\bar{U})$ and $\mathscr{T}_{\mu}(\bar{U})$.

We will prove this theorem in Sect. IV.

\section{Toeplitz Operators}

Our goal in this section is to prove Theorem III.5. The technique to achieve this is a detailed analysis of the structure of the $\mathbb{C}^{*}$-algebra $\mathscr{T}_{\mu}(\bar{U})$. Our analysis follows the standard methods (in particular those of [10,1 and 6]). For the reader's convenience we include the details of the proofs referring to the literature at appropriate places. Throughout this section, $f$ denotes a continuous function on $\bar{U}$ and $\|f\|_{\infty}$ denotes its sup-norm.

Lemma IV.1. (i) $\left\|T_{f}\right\| \leqq\|f\|_{\infty}$,

(ii) $T_{f}=0$, if and only if $f=0$. 
Proof. Part (i) is clear. To prove part (ii) we observe that $T_{f}=0$ if and only if $\left(\varphi_{m}, T_{f} \varphi_{n}\right)=0, m, n=0,1,2, \ldots$, where $\varphi_{n}$ is defined by (III.10). Since

$$
\left(\zeta^{m}, T_{f} \zeta^{n}\right)=\int_{U} \zeta^{m \bar{\zeta}^{n}} f(\zeta) d \mu_{r}(\zeta)
$$

and since the monomials $\zeta^{m} \overline{\zeta^{n}}$ generate a dense subspace in $L^{2}\left(U, d \mu_{r}\right)$ this is possible if and only if $f=0$.

Let $\mathscr{K}\left(\mathscr{H}_{U}\right)$ denote the $\mathbb{C}^{*}$-algebra of compact operators on $\mathscr{H}_{U}$.

Lemma IV.2. $T_{f} \in \mathscr{K}\left(\mathscr{H}_{U}\right)$ if and only iff $\uparrow_{\partial U}=0$.

Proof. We follow [1] and [6]. Suppose that $f \uparrow_{\partial U}=0$. There is a sequence $\left\{f_{n}\right\}$ of continuous functions such that $\operatorname{supp} f_{n} \subset U$ and $\left\|f-f_{n}\right\|_{\infty} \rightarrow 0$. Let $\left\{\varphi_{m}\right\}$ be a sequence in $\mathscr{H}_{U}$ such that $\varphi_{m} \rightarrow 0$ weakly (recall that a weakly convergent sequence is bounded). We claim that $T_{f} \varphi_{m} \rightarrow 0$ in norm which means that $T_{f}$ is compact. Indeed, since $\varphi_{m}(\zeta) \rightarrow 0$ pointwise, it follows that, for a fixed $n, \varphi_{m}(\zeta) \rightarrow 0$ uniformly on $\operatorname{supp} f_{n}$. Therefore,

$$
\begin{aligned}
\left\|T_{f} \varphi_{m}\right\| & \leqq\left\|T_{f_{n}} \varphi_{m}\right\|+\left\|T_{f-f_{n}} \varphi_{m}\right\| \\
& \leqq C\left(\sup _{\zeta \in \operatorname{supp} f_{n}}\left|\varphi_{m}(\zeta)\right|\left\|f_{n}\right\|+\left\|f-f_{n}\right\|_{\infty}\left\|\varphi_{m}\right\|\right),
\end{aligned}
$$

where $C=\left\{\int_{U} d \mu_{r}(\zeta)\right\}^{1 / 2}<\infty$. Choosing $n$ sufficiently large and using the fact that $\left\|\varphi_{m}\right\| \leqq K$, we see that the right-hand side of this inequality is arbitrarily small.

To prove the converse we consider the sequence of elements of $\mathscr{H}_{U}$

$$
\varphi_{n}(\zeta):=K\left(\zeta, \eta_{n}\right) / K\left(\eta_{n}, \eta_{n}\right)^{1 / 2}
$$

where $\eta_{n} \in U, \eta_{n} \rightarrow \eta \in \partial U$. Then $\left\|\varphi_{n}\right\|=1$ and $\varphi_{n}(\zeta) \rightarrow 0$ uniformly outside a neighborhood $V$ of $\eta$. Passing to a subsequence, if necessary, we may assume that $\varphi_{n} \rightarrow 0$ weakly. Since

$$
\begin{aligned}
\left\|\left(T_{f}-f(\eta)\right) \varphi_{n}\right\|^{2} & \leqq \int_{U}|f(\zeta)-f(\eta)|^{2}\left|\varphi_{n}(\zeta)\right|^{2} d \mu_{r}(\zeta) \\
& \leqq\left\{\sup _{\zeta \in V}|f(\zeta)-f(\eta)|\right\}^{2}+\left\{\sup _{\zeta \in U \backslash V}\left|\varphi_{n}(\zeta)\right|\right\}^{2}\|f-f(\eta)\|^{2},
\end{aligned}
$$

we see that $\left\|\left(T_{f}-f(\eta)\right) \varphi_{n}\right\| \rightarrow 0$. As a consequence,

$$
|f(\eta)|=\left\|f(\eta) \varphi_{n}\right\| \leqq\left\|T_{f} \varphi_{n}\right\|+\left\|\left(T_{f}-f(\eta)\right) \varphi_{n}\right\| \rightarrow 0,
$$

since $T_{f}$ is compact. Therefore, $f(\eta)=0$.

Lemma IV.3. $T_{f} T_{g}-T_{f g},\left[T_{f}, T_{g}\right] \in \mathscr{K}\left(\mathscr{H}_{U}\right)$.

Proof. We follow [1]. Obviously, the second claim is a consequence of the first, so we need prove that $T_{f} T_{g}-T_{f g} \in \mathscr{K}\left(\mathscr{H}_{U}\right)$. Clearly $T_{f g}-T_{f} T_{g}=P M_{f}(1-P) M_{g}$. Let $\mathscr{H}_{U}^{\perp}$ denote the orthogonal complement of $\mathscr{H}_{U}$ in $L^{2}\left(U, d \mu_{r}\right)$ and let $H_{f}:=$ $(1-P) M_{f} P: \mathscr{H}_{U} \rightarrow \mathscr{H}_{U}^{\frac{1}{U}}$. Then

$$
T_{f g}-T_{f} T_{g}=H_{f}^{*} H_{g}
$$


Furthermore,

$$
H_{f g}=S_{f} H_{g}+H_{f} T_{g},
$$

where $S_{f}: \mathscr{H}_{U}^{\perp} \rightarrow \mathscr{H}_{U}^{\perp}$ is defined by $S_{f}:=(1-P) M_{f}$. Let now $\mathscr{B}:=\left\{f \in C(\bar{U}): H_{f}\right.$ is compact $\}$. As a consequence of (IV.2), $\mathscr{B}$ is a closed subalgebra of $C(\bar{U})$. Also, $H_{1}=0$, $H_{\zeta}=0$ and by (IV.1),

$$
H_{\zeta}^{*} H_{\bar{\zeta}}=T_{\zeta \bar{\zeta}}-T_{\zeta} T_{\bar{\zeta}}=[\pi(z), \pi(\bar{z})] \in \mathscr{K}\left(\mathscr{H}_{U}\right) .
$$

So $1, \zeta, \bar{\zeta} \in \mathscr{B}$, and thus by the Stone-Weierstrass theorem $\mathscr{B}=C(\bar{U})$.

Let $J \subset \mathscr{T}_{\mu}(\bar{U})$ be the commutator ideal of $\mathscr{T}_{\mu}(\bar{U})$, i.e., the smallest norm-closed, two sided ideal in $\mathscr{T}_{\mu}(\bar{U})$ containing all the commutators.

Lemma IV.4. $J=\mathscr{K}\left(\mathscr{H}_{U}\right)$.

Proof. We follow [1]. To show that $\mathscr{K}\left(\mathscr{H}_{U}\right) \subset \mathscr{T}_{\mu}\left(\mathscr{H}_{U}\right)$ we use Theorem 5.39 in [10]. Firstly, $\mathscr{K}\left(\mathscr{H}_{U}\right) \cap \mathscr{T}_{\mu}\left(\mathscr{H}_{U}\right) \neq \varnothing$, as $\left[T_{\zeta}, T_{\bar{\zeta}}\right] \in \mathscr{K}\left(\mathscr{H}_{U}\right)$. Secondly, we claim that $\mathscr{T}_{\mu}(\bar{U})$ is irreducible. Indeed, let $Q$ be an orthogonal projection in $\mathscr{H}_{U}$ which commutes with $\mathscr{T}_{\mu}(\bar{U})$. Then $(Q \varphi)(\zeta)=Q T_{\varphi(\bar{\zeta})} 1=\varphi(\zeta)(Q 1)(\zeta)$ for all polynomials $\varphi \in \mathscr{H}_{U}$. From $Q^{2}=Q$ we have $Q 1=1$ and the claim follows. Therefore, by the quoted theorem, $\mathscr{K}\left(\mathscr{H}_{U}\right) \subset \mathscr{T}_{\mu}(\widetilde{U})$. As a consequence of Lemma IV.3, $J \subset \mathscr{K}\left(\mathscr{H}_{U}\right)$. Since $\mathscr{K}\left(\mathscr{H}_{U}\right)$ is simple $[10], \mathscr{K}\left(H_{U}\right)=J$.

Theorem IV.5. (i) There is a short exact sequence of $\mathbb{C}^{*}$-algebra (the Brown-DouglasFillmore sequence)

$$
0 \rightarrow \mathscr{K}\left(\mathscr{H}_{U}\right) \rightarrow \mathscr{T}_{\mu}(\bar{U}) \rightarrow C(\partial U) \rightarrow 0 .
$$

(ii) Every element $A \in \mathscr{T}_{\mu}(\bar{U})$ can be written as

$$
A=T_{f}+K
$$

where $K \in \mathscr{K}\left(\mathscr{H}_{U}\right)$. Furthermore,

$$
\left\|T_{f}+K\right\| \geqq \sup _{|\zeta|=1}|f(\zeta)| .
$$

Proof. (i) We claim that

$$
\mathscr{T}_{\mu}(\bar{U}) / \mathscr{K}\left(\mathscr{H}_{U}\right) \cong C(\partial U)
$$

as $\mathbb{C}^{*}$-algebras. Indeed, let $\sigma: C(\bar{U}) \rightarrow \mathscr{T}_{\mu}(U) / \mathscr{K}\left(\mathscr{H}_{U}\right)$ be the map defined by $\sigma(f):=T_{f}\left(\bmod \mathscr{K}\left(\mathscr{H}_{U}\right)\right)$. By Lemma IV.3, $\sigma$ is a $*$-homomorphism whose range is dense. By Lemma IV.2, $\operatorname{Ker}(\sigma)=C^{(0)}(\bar{U}):=\left\{f \in C(\bar{U}): f \uparrow_{\partial U}=0\right\}$. So $\sigma$ induces an injective $*$-homomorphism

$$
\tilde{\sigma}: C(\bar{U}) / C^{(0)}(\bar{U}) \cong C(\partial U) \rightarrow \mathscr{T}_{\mu}(\bar{U}) / \mathscr{K}\left(\mathscr{H}_{U}\right) .
$$

By Proposition 4.67 in [10], $\tilde{\sigma}$ is an isometry, so $\operatorname{Ran}(\sigma)=\operatorname{Ran}(\tilde{\sigma})=\mathscr{T}_{\mu}(\bar{U}) / \mathscr{K}\left(\mathscr{H}_{U}\right)$, and $\tilde{\sigma}$ is an isomorphism of $\mathbb{C}^{*}$-algebras.

(ii) As a consequence of (i), every element in $\mathscr{T}_{\mu}(\bar{U})$ is of the form (IV.4). Since $j: \mathscr{T}_{\mu}(\bar{U}) \rightarrow C(\partial U), j\left(T_{f}+K\right):=f \uparrow \partial U$ is a $*$-monomorphism of $\mathbb{C}^{*}$-algebras, it follows from Proposition 4.67 in $[10]$ that $\sup |f(\zeta)| \leqq\left\|T_{f}+K\right\|$.

Now we are in a position to prove Theorem III.5. 
Proof of Theorem III.5. Let

$$
a=\sum_{\substack{k_{1}, \ldots, k_{n} \\ l_{1}, \ldots, l_{n}}} \alpha_{k l} z^{k_{1}} \bar{z}^{l_{1}} \cdots z^{k_{n}} \bar{z}^{l_{n}} \in P_{\mu}(\bar{U}),
$$

where $\alpha_{k l} \in \mathbb{C}$. By (II.22) and Theorems III.2 and II.4,

$$
\|a\|=\max \left\{\left\|\pi\left(\sum_{k, l} \alpha_{k l} z^{k_{1}} \bar{z}^{l_{1}} \cdots z^{k_{n}} \bar{z}^{l_{n}}\right)\right\|, \sup _{|\zeta|=1}\left|\sum_{k, l} \alpha_{k l} \zeta^{k_{1}+\cdots+k_{n}} \bar{\zeta}^{l_{1}+\cdots+l_{n}}\right|\right\} \text {. }
$$

By (IV.5), the maximum is achieved at $\left\|\pi\left(\sum_{k, l} \alpha_{k l} z^{k_{1}} \bar{z}^{l_{1}} \cdots z^{k_{n}} \bar{z}^{l_{n}}\right)\right\|$. Therefore $\|a\|=$ $\|\pi(a)\|$. The claim follows.

As a consequence of Theorems III.5 and IV.5 we obtain:

Corollary IV.6. The $\mathbb{C}^{*}$-algebra $C_{\mu}(\bar{U})$ is a GCR algebra.

Let now $\mathscr{H}=l^{2}\left(\mathbb{Z}_{+}\right)$and let $\left\{f_{n}\right\}_{n \geqq 0}$ be an orthonormal basis for $\mathscr{H}$. The operator $S$ defined by $S f_{n}:=f_{n+1}, n \geqq 0$, is called the unilateral shift [10]. Let $\mathbb{C}^{*}(S)$ be the unital $\mathbb{C}^{*}$-algebra generated by $S$.

Theorem IV.7. $C_{\mu}(\bar{U})$ is isomorphic with $\mathbb{C}^{*}(S)$.

Proof. Let $E_{m, n} \in \mathscr{L}(\mathscr{H})$ be defined by $E_{m, n} f_{p}:=\delta_{n p} f_{m}$. Then $S=\sum_{n \geqq 0} E_{n+1, n}$ and $\pi(\bar{z})=\sum_{n \geqq 0}\{(n+1) \mu /(1+(n+1) \mu)\}^{1 / 2} E_{n+1, n}$. It is clear that $S-\pi(\bar{z}) \in \mathscr{K}(\mathscr{H})$. Consequently, $\mathbb{C}^{*}(S) \subset \pi\left(C_{\mu}(\bar{U})\right) \cong C_{\mu}(\bar{U})$. On the other hand, since $S+K \in \mathbb{C}^{*}(S)$ (see $[10])$, it follows that $\pi(\bar{z}) \in \mathbb{C}^{*}(S)$, i.e., $\pi\left(C_{\mu}(\bar{U})\right) \subset \mathbb{C}^{*}(S)$.

\section{The Action of $S U(1,1)$}

In Sect. II we constructed a group action $S U(1,1) \rightarrow \operatorname{Aut}\left(C_{\mu}(\bar{U})\right)$. Under the identification $C_{\mu}(\bar{U}) \cong \mathscr{T}_{\mu}(\bar{U})$, this action can be implemented by a projective unitary representation of $S U(1,1)$ on $\mathscr{H}_{U}$.

For $\lambda \in \mathbb{C}^{*}:=\mathbb{C} \backslash\{0\}$ we set $\log \lambda:=\log |\lambda|+i \arg \lambda$, where $-\pi<\arg \lambda \leqq \pi$. If $\gamma \in S U(1,1)$, we let $a_{\gamma}$ and $b_{\gamma}$ denote the matrix elements of $\gamma^{-1}$ as in (II.11). The function [14]

$$
\frac{1}{2 \pi i}\left\{\log \left(\bar{b}_{\gamma_{2}} \gamma_{1}^{-1} \zeta+\bar{a}_{\gamma_{2}}\right)-\log \left(\bar{b}_{\gamma_{1} \gamma_{2}} \zeta+\bar{a}_{\gamma_{1} \gamma_{2}}\right)+\log \left(\bar{b}_{\gamma_{1}} \zeta+\bar{a}_{\gamma_{1}}\right)\right\}
$$

where $\gamma_{1}, \gamma_{2} \in S U(1,1), \zeta \in U$, is independent of $\zeta$ (to see this, differentiate with respect to $\zeta)$. We denote the value of (V.1) by $\lambda\left(\gamma_{1}, \gamma_{2}\right)$. Furthermore, setting $\zeta=0$ it is easy to see that $\lambda\left(\gamma_{1}, \gamma_{2}\right) \in\{-1,0,1\}$. In fact, the function $\lambda: S U(1,1) \times S U(1,1) \rightarrow \mathbb{Z}$ defines a cohomology class in $H^{2}(S U(1,1), \mathbb{Z})$, i.e.,

$$
\lambda\left(\gamma_{2}, \gamma_{3}\right)-\lambda\left(\gamma_{1} \gamma_{2}, \gamma_{3}\right)+\lambda\left(\gamma_{1}, \gamma_{2} \gamma_{3}\right)-\lambda\left(\gamma_{1}, \gamma_{2}\right)=0
$$

(to see this, use the fact that (V.1) is independent of $\zeta$ ). We set

$$
\sigma\left(\gamma_{1}, \gamma_{2}\right):=\exp \left\{2 \pi i r \lambda\left(\gamma_{1}, \gamma_{2}\right)\right\}
$$


For $\gamma \in S U(1,1)$ and $\varphi \in \mathscr{H}_{U}$ we define

$$
\left(U_{\gamma} \varphi\right)(\zeta):=\left(\bar{b}_{\gamma} \zeta+\bar{a}_{\gamma}\right)^{-r} \varphi\left(\gamma^{-1} \zeta\right)
$$

where $\left(\bar{b}_{\gamma} \zeta+\bar{a}_{\gamma}\right)^{-r}:=\exp \left\{-r \log \left(\bar{b}_{\gamma} \zeta+\bar{a}_{\gamma}\right)\right\}$. Then $U_{\gamma}$ is a unitary operator on $\mathscr{H}_{U}$, and

$$
U_{\gamma_{1} \gamma_{2}}=\sigma\left(\gamma_{1}, \gamma_{2}\right) U_{\gamma_{1}} U_{\gamma_{2}} .
$$

Therefore, as a consequence of (V.2), $\gamma \rightarrow U_{\gamma}$ is a projective unitary representation of $S U(1,1)$.

Theorem V.1. The $S U(1,1)$ action $\gamma \rightarrow \rho_{\gamma}$ on $C_{\mu}(\bar{U})$ is implemented by $\gamma \rightarrow U_{\gamma}$.

Proof. We need verify that

$$
\rho_{\gamma}(\bar{z})=U_{\gamma} \bar{z} U_{\gamma-1}
$$

Indeed, from (III.11),

$$
\begin{aligned}
\left(U_{\gamma} \bar{z} U_{\gamma^{-1}} \varphi\right)(\zeta) & =(\bar{b} \zeta+\bar{a})^{-r}\left(\bar{z} U_{\gamma^{-1}} \varphi\right)\left(\gamma^{-1} \zeta\right)=(\bar{b} \zeta+\bar{a})^{-r} \frac{a \zeta+b}{\bar{b} \zeta+\bar{a}}\left(U_{\gamma^{-1}} \varphi\right)\left(\gamma^{-1} \zeta\right) \\
& =(\bar{b} \zeta+\bar{a})^{-r} \frac{a \zeta+b}{\bar{b} \zeta+\bar{a}}\left(-\bar{b} \gamma^{-1} \zeta+a\right)^{-r} \varphi(\zeta)=\frac{a \zeta+b}{\bar{b} \zeta+\bar{a}} \varphi(\zeta)=\rho_{\gamma}(\bar{z}) \varphi(\zeta)
\end{aligned}
$$

as claimed.

\section{Asymptotics of Products of Toeplitz Operators}

In this section we show that, in a suitable sense, $C_{\mu}(U)$ is a quantum deformation of the unit disc. To be more specific, we prove that for smooth $f$ and $g, \frac{1}{\mu}\left[T_{f}, T_{g}\right]$
approaches its classical value, as $\mu \rightarrow 0$.

Let $C^{\infty}(\bar{U})$ denote the Fréchet space of smooth functions on $U$ whose derivatives extend to continuous functions on $\bar{U}$. For $f, g \in C^{\infty}(\bar{U})$ we define

$$
\{f, g\}(\zeta):=i\left(1-|\zeta|^{2}\right)^{2}[\partial f(\zeta) \bar{\partial} g(\zeta)-\bar{\partial} f(\zeta) \partial g(\zeta)]
$$

the Poisson bracket of $f$ and $g$ corresponding to the Poincare symplectic form on $U, \omega:=\frac{i}{2}\left(1-|\zeta|^{2}\right)^{-2} d \zeta \wedge d \bar{\zeta}$. Obviously, $\{f, g\} \in C^{\infty}(\bar{U})$. The goal of this section is to prove the following theorems.

Theorem VI.1. Let $f \in C(\bar{U})$. Then

$$
\left\|T_{f}\right\| \leqq\|f\|_{\infty} \leqq\left\|T_{f}\right\|+o(1)
$$

as $r \rightarrow \infty$. In particular, $\lim _{r \rightarrow \infty}\left\|T_{f}\right\|=\|f\|_{\infty}$.

Theorem VI.2. Let $f, g \in C^{\infty}(\bar{U})$ and let $r$ be sufficiently large. Then:

(i) There exists a constant $C$ (depending on $f$ and $g$ ) such that

$$
\left\|r\left(T_{f} T_{g}-T_{f g}\right)+T_{\left(1-|\zeta|^{2}\right)^{2} \partial f \bar{\partial} g}\right\| \leqq C r^{-1 / 2} .
$$


(ii) There is a constant $C$ (depending on $f$ and $g$ ) such that

$$
\left\|\frac{r}{i}\left[T_{f}, T_{g}\right]-T_{\{f, g\}}\right\| \leqq C r^{-1 / 2} .
$$

Remark. It should be remembered that the operators and the operator norms in (VI.2-4) depend on $r$ even though, for the sake of notational simplicity, this is not explicit in our formulas. Estimate (VI.2) says that $\lim _{\mu \rightarrow 0}\left\|T_{f}\right\|=\|f\|$, while estimate (IV.4) says that $\lim _{\mu \rightarrow 0}\left\|\frac{1}{i \mu}\left[T_{f}, T_{g}\right]-T_{\{f, g\}}\right\|=0$. This shows that $C_{\mu}(U)$ is a quantum deformation of the unit disc in a sense close to Rieffel's "strict deformation quantization" [17].

Proof of Theorem VI.1. The first inequality in (VI.2) is clear. To prove the second one, we define $\gamma_{\zeta} \in S U(1,1), \zeta \in U$, by

$$
\gamma_{\zeta}=\left(1-|\zeta|^{2}\right)^{-1 / 2}\left(\begin{array}{cc}
1 & \zeta \\
\bar{\zeta} & 1
\end{array}\right)
$$

Observe that $\gamma_{\zeta}(0)=\zeta$, and

$$
\left|\gamma_{\zeta}(\eta)-\gamma_{\zeta}(0)\right| \leqq \frac{|\eta|}{1-|\eta|}
$$

In particular, if $|\eta|<\delta \leqq 1 / 2$, then

$$
\left|\gamma_{\zeta}(\eta)-\gamma_{\zeta}(0)\right| \leqq 2 \delta
$$

Lemma VI.3. If $f \in C(\bar{U})$, then

$$
\sup _{\zeta \in U}\left|f(\zeta)-(r-1) \int_{U} f\left(\gamma_{\zeta}(\eta)\right) d \mu_{r}(\eta)\right|=o(1)
$$

as $r \rightarrow \infty$.

We shall prove this lemma after completing the main line of the argument. Using the reproducing property of the Bergman kernel, we write

$$
\begin{aligned}
f(\zeta) & =(r-1) \int_{U} \gamma_{\zeta}^{*} f(\eta) d \mu_{r}(\eta)+\left(f(\zeta)-(r-1) \int_{U} \gamma_{\zeta}^{*} f(\eta) d \mu_{r}(\eta)\right) \\
& =(r-1) \int_{U^{2}} K(\theta, \eta) \gamma_{\zeta}^{*} f(\eta) d \mu_{r}(\theta)+\left(f(\zeta)-(r-1) \int_{U} \gamma_{\zeta}^{*} f(\eta) d \mu_{r}(\eta)\right) \\
& =\left(T_{\gamma_{\zeta}^{*} f} \varphi_{0}, \varphi_{0}\right)+\left(f(\zeta)-(r-1) \int_{U} \gamma_{\zeta}^{*} f(\eta) d \mu_{r}(\eta)\right)
\end{aligned}
$$

where $\gamma_{\zeta}^{*} f(\eta):=f\left(\gamma_{\zeta}(\eta)\right)$, and where $\varphi_{0}$ is given by (III.10). As a consequence of Lemma VI.3,

$$
\sup _{\zeta \in U}|f(\zeta)| \leqq \sup _{\zeta \in U}\left\|T_{\gamma_{\zeta}^{*} f}\right\|+o(1)
$$

But, $T_{\gamma_{\zeta}^{*} f}=U_{\gamma \zeta} T_{f} U_{\gamma_{\zeta}}^{-1}$, with $U_{\gamma_{\zeta}}$ unitary, and so $\|f\|_{\infty} \leqq\left\|T_{f}\right\|+o(1)$, as claimed. 
Proof of Lemma VI.3. Since $f$ is continuous on a compact set, it is uniformly continuous. Given $\varepsilon>0$, we take $\delta \leqq 1 / 2$ so that $\left|f(\eta)-f\left(\eta^{\prime}\right)\right|<\varepsilon / 2$, whenever $\left|\eta-\eta^{\prime}\right|<2 \delta$. Then,

$$
\begin{aligned}
f(\eta)-(r-1) \int_{U} f\left(\gamma_{\zeta}(\eta)\right) d \mu_{r}(\eta) & =(r-1) \int_{U}\left(f\left(\gamma_{\zeta}(0)\right)-f\left(\gamma_{\zeta}(\eta)\right)\right) d \mu_{r}(\eta) \\
& =\int_{|\eta|<\delta} \cdots+\int_{\delta<|\eta|<1} \cdots:=I_{1}+I_{2} .
\end{aligned}
$$

Now, using (VI.6),

$$
\left|I_{1}\right| \leqq \sup _{|\eta|<\delta}\left|f\left(\gamma_{\zeta}(0)\right)-f\left(\gamma_{\zeta}(\eta)\right)\right|<\varepsilon / 2
$$

Furthermore,

$$
\left|I_{2}\right| \leqq 2\|f\|_{\infty} \int_{\delta<|\eta|<1} d \mu_{r}(\eta)=2\|f\|_{\infty}\left(1-\delta^{2}\right)^{r-1},
$$

which is less than $\varepsilon / 2$, for $r$ sufficiently large.

Proof of Theorem VI.2. Obviously, (ii) is a consequence of (i) and (VI.1). To prove (i) we write

$$
\left(T_{f} T_{g} \varphi, \psi\right)=\int_{U^{2}} K(\zeta, \eta) f(\zeta) g(\eta) \varphi(\eta) \overline{\psi(\zeta)} d \mu_{r}(\zeta) d \mu_{r}(\eta)
$$

where $\varphi, \psi \in \mathscr{H}_{U}$. In the $\eta$-integral we substitute $\eta=\gamma_{\zeta} \theta$, where $\gamma_{\zeta} \in S U(1,1)$ is defined by (VI.5). Then

$$
\left(T_{f} T_{g} \varphi, \psi\right)=\pi^{-1}(r-1) \int_{U^{2}} f(\zeta) g\left(\gamma_{\zeta} \theta\right)\left(U_{\gamma_{\zeta}^{-1}} \varphi\right)(\theta) \overline{\psi(\zeta)}\left(1-|\zeta|^{2}\right)^{-r / 2} d \mu_{r}(\zeta) d \mu_{r}(\theta)
$$

This form of (VI.8) is suitable for asymptotic analysis. From Taylor's theorem,

$$
\begin{aligned}
g\left(\gamma_{\zeta} \theta\right)= & g(\zeta)+\left(1-|\zeta|^{2}\right) \partial g(\zeta) \theta+\left(1-|\zeta|^{2}\right) \bar{\partial} g(\zeta) \bar{\theta} \\
& +\left(1-|\zeta|^{2}\right)\left[-\bar{\zeta} \partial g(\zeta)+\frac{1}{2}\left(1-|\zeta|^{2}\right) \partial^{2} g(\zeta)\right] \theta^{2} \\
& +\left(1-|\zeta|^{2}\right)\left[-\zeta \bar{\partial} g(\zeta)+\frac{1}{2}\left(1-|\zeta|^{2}\right) \bar{\partial}^{2} g(\zeta)\right] \bar{\theta}^{2} \\
& +\left(1-|\zeta|^{2}\right)^{2} \partial \bar{\partial} g(\zeta) \theta \bar{\theta}+G(\theta, \zeta),
\end{aligned}
$$

where $G(\theta, \zeta)$ is the second order remainder. Let us assume for a moment that the following fact is true.

Lemma IV.4. There is a constant $C$ (depending on $f$ and $g$ ) such that

$$
\begin{aligned}
& \left|\pi^{-1}(r-1) \int_{U^{2}} f(\zeta) G(\theta, \zeta)\left(U_{\gamma_{\zeta}^{-1}} \varphi\right)(\theta) \overline{\psi(\zeta)}\left(1-|\zeta|^{2}\right)^{-r / 2} d \mu_{r}(\zeta) d \mu_{r}(\theta)\right| \\
& \quad \leqq C r^{-3 / 2}\|\varphi\|\|\psi\| .
\end{aligned}
$$

Substituting (VI.10) into (VI.9) and using the fact that $\theta \rightarrow\left(U_{\gamma_{\xi}^{-1}} \varphi\right)(\theta)$ is a holomorphic function we find:

$$
\begin{aligned}
\left(T_{f} T_{g} \varphi, \psi\right)= & \int_{U} f(\zeta) g(\zeta) \varphi(\zeta) \overline{\psi(\zeta)} d \mu_{r}(\zeta)+\pi^{-1}(r-1) \\
& \cdot \int_{U^{2}} f(\zeta) \bar{\partial} g(\zeta) \frac{\partial}{\partial \theta}\left(U_{\gamma_{\zeta}^{-1}} \varphi\right)(0) \overline{\psi(\zeta)}\left(1-|\zeta|^{2}\right)^{-r / 2+1}|\theta|^{2} d \mu_{r}(\zeta) d \mu_{r}(\theta)
\end{aligned}
$$




$$
\begin{aligned}
& +\pi^{-1}(r-1) \int_{U^{2}} f(\zeta)\left(1-|\zeta|^{2}\right)^{2} \partial \bar{\partial} g(\zeta) \varphi(\zeta) \overline{\psi(\zeta)}|\theta|^{2} d \mu_{r}(\zeta) d \mu_{r}(\theta) \\
& +\pi^{-1}(r-1) \int_{U^{2}} f(\zeta)\left[-\zeta \bar{\partial} g(\zeta)+\frac{1}{2}\left(1-|\zeta|^{2}\right) \bar{\partial}^{2} g(\zeta)\right] \frac{\partial^{2}}{\partial \theta^{2}}\left(U_{\gamma_{\zeta}^{-1}} \varphi\right)(0) \\
& \cdot \overline{\psi(\zeta)}\left(1-|\zeta|^{2}\right)^{-r / 2+1}|\theta|^{4} d \mu_{r}(\zeta) d \mu_{r}(\theta)+R
\end{aligned}
$$

where $R$ denotes the remainder term which obeys the estimate (VI.11). Observe that the first term on the right-hand side is equal to $\left(T_{f g} \varphi, \psi\right)$. Furthermore, using

and

$$
\pi^{-1}(r-1) \int_{U}|\theta|^{2} d \mu_{r}(\theta)=r^{-1}
$$

$$
\left(1-|\zeta|^{2}\right)^{-r / 2} \frac{\partial}{\partial \theta}\left(U_{\gamma_{\zeta}^{-1}} \varphi\right)(\theta)=\left(1-|\zeta|^{2}\right)^{-r+1}(1+\bar{\zeta} \theta)^{-1} \frac{\partial}{\partial \zeta}\left\{\left(1-|\zeta|^{2}\right)^{r / 2}\left(U_{\gamma_{\zeta}^{-1}} \varphi\right)(\theta)\right\}
$$

we write the second and third terms in (VI.12) as

$$
\begin{aligned}
& \frac{1}{r} \int_{U} f(\zeta) \bar{\partial} g(\zeta) \overline{\psi(\zeta)}\left(1-|\zeta|^{2}\right)^{-r+2} \frac{\partial}{\partial \zeta}\left\{\left(1-|\zeta|^{2}\right)^{r} \varphi(\zeta)\right\} d \mu_{r}(\zeta) \\
& \quad+\frac{1}{r} \int_{U} f(\zeta)\left(1-|\zeta|^{2}\right)^{2} \partial \bar{\partial} g(\zeta) \varphi(\zeta) \overline{\psi(\zeta)} d \mu_{r}(\zeta) .
\end{aligned}
$$

Integrating by parts we see that this sum is equal to

$$
-\frac{1}{r} \int\left(1-|\zeta|^{2}\right)^{2} \partial f(\zeta) \bar{\partial} g(\zeta) \varphi(\zeta) \overline{\psi(\zeta)} d \mu_{r}(\zeta)=-\frac{1}{r}\left(T_{\left(1-|\zeta|^{2}\right)^{2} \partial f \bar{\partial} g} \varphi, \psi\right) .
$$

We claim that the fourth term in (VI.12) can be estimated by $\mathrm{Cr}^{-2}\|\varphi\|\|\psi\|$, with $C$ independent of $r$. We proceed as follows. Using (VI.13) twice, we find that

$$
\begin{aligned}
\left(1-|\zeta|^{2}\right)^{-r / 2} \frac{\partial^{2}}{\partial \theta^{2}}\left(U_{\gamma_{\zeta}^{-1}} \varphi\right)(0)= & -\left(1-|\zeta|^{2}\right)^{-r+1} \bar{\zeta} \frac{\partial}{\partial \zeta}\left\{\left(1-|\zeta|^{2}\right)^{r} \varphi(\zeta)\right\} \\
& +\left(1-|\zeta|^{2}\right)^{-r+1} \frac{\partial}{\partial \zeta}\left\{\left(1-|\zeta|^{2}\right) \frac{\partial}{\partial \zeta}\left[\left(1-|\zeta|^{2}\right)^{r} \varphi(\zeta)\right]\right\} .
\end{aligned}
$$

We substitute this into (VI.12) and use the fact that

$$
\pi^{-1}(r-1) \int_{U}|\theta|^{4} d \mu_{r}(\theta)=\frac{2}{r(r+1)} .
$$

Integrating by parts we write the integral as

$$
\frac{1}{r(r+1)} \int_{U} F(\zeta) \varphi(\zeta) \overline{\psi(\zeta)} d \mu_{r}(\zeta)
$$

with $F(\zeta)$ continuous and bounded on $U$. Finally, we estimate (VI.15) by $r^{-2} \sup _{\zeta \in U}|F(\zeta)|\|\varphi\|\|\psi\|$. This concludes the proof of (VI.3) up to the proof of Lemma VI.4. 
Proof of Lemma VI.4. We claim that $G(\theta, \zeta)$ can be written as

$$
G(\theta, \zeta)=\sum_{0 \leqq j \leqq 3} g_{j}(\theta, \zeta) \theta^{j} \bar{\theta}^{3-j}
$$

where $g_{j}, 0 \leqq j \leqq 3$, are smooth functions on $U \times U$ with the following properties:

(i) $\left|g_{j}(\theta, \zeta)\right| \leqq O(1)\left(1-|\zeta|^{2}\right)(1-|\theta|)^{-6}$,

(ii) $\left|\frac{\partial}{\partial \zeta}\left\{\left(1-|\zeta|^{2}\right)^{-1} g_{j}(\theta, \zeta)\right\}\right|+\left|\frac{\partial}{\partial \bar{\zeta}}\left\{\left(1-|\zeta|^{2}\right)^{-1} g_{j}(\theta, \zeta)\right\}\right| \leqq O(1)(1-|\theta|)^{-8}$,

(iii) $\left|\frac{\partial}{\partial \theta} g_{j}(\theta, \zeta)\right|+\left|\frac{\partial}{\partial \bar{\theta}} g_{j}(\theta, \zeta)\right| \leqq O(1)\left(1-|\zeta|^{2}\right)(1-|\theta|)^{-8}$.

Indeed, we write

$$
\begin{aligned}
G(\theta, \zeta) & \left.=\frac{1}{2} \int_{0}^{1}(1-s)^{2} \frac{d^{3}}{d s^{3}} \gamma_{\zeta}^{*} g(s \theta)\right) d s \\
& =\frac{1}{2} \sum_{0 \leqq j \leqq 3}\left(\begin{array}{l}
3 \\
j
\end{array}\right) \int_{0}^{1}(1-s)^{2} \partial_{s \theta}^{j} \bar{\partial}_{s \theta}^{3-j} \gamma_{\zeta}^{*} g(s \theta) d s \theta^{j} \bar{\theta}^{3-j},
\end{aligned}
$$

and define

$$
g_{j}(\theta, \zeta):=\frac{1}{2}\left(\begin{array}{c}
3 \\
j
\end{array}\right) \int_{0}^{1}(1-\zeta)^{2} \partial_{s \theta}^{j} \bar{\partial}_{s \theta}^{3-j} \gamma_{\zeta}^{*} g(s \theta) d s
$$

To verify that $g_{j}(\theta, \zeta)$ obeys (VI.17)-(VI.19) we carry out the differentiations in (VI.20) according to the chain rule. Using the fact that for $k \geqq 1$

$$
\frac{\partial^{k}}{\partial \theta^{k}} \gamma_{\zeta}(\theta)=(-1)^{k-1} k !\left(1-|\zeta|^{2}\right) \bar{\zeta}^{k-1}(1+\bar{\zeta} \theta)^{-k-1}
$$

as well as the inequality

$$
|1+s \bar{\zeta} \theta|^{-1} \leqq(1-s|\theta|)^{-1} \leqq(1-|\theta|)^{-1}, \quad 0 \leqq s \leqq 1,
$$

we easily obtain the bound

$$
\left|g_{j}(\theta, \zeta)\right| \leqq O(1)\left\{\sum_{1 \leqq \alpha+\beta \leqq 3}\left\|\partial^{\alpha} \bar{\partial}^{\beta} g\right\|_{\infty}\right\}\left(1-|\zeta|^{2}\right)(1-|\theta|)^{-6},
$$

which proves (VI.17). Inequalities (VI.18) and (VI.19) follow from an analogous argument for the derivatives of (VI.20).

Let us now estimate the contribution that each term in (VI.16) gives to (VI.11). Let $j=0$. Using

$$
\bar{\theta}\left(1-|\theta|^{2}\right)^{r-2}=\frac{1}{r-1} \frac{\partial}{\partial \theta}\left(1-|\theta|^{2}\right)^{r-1}
$$

and integrating by parts with respect to $\theta$ we find that

$$
\pi^{-1}(r-1) \int_{U^{2}} f(\zeta) g_{0}(\theta, \zeta) \bar{\theta}^{3}\left(U_{\gamma_{\zeta}^{-1}} \varphi\right)(\theta) \overline{\psi(\zeta)}\left(1-|\zeta|^{2}\right)^{-r / 2} d \mu_{r}(\zeta) d \mu_{r}(\theta)
$$




$$
\begin{aligned}
= & -\pi^{-1} \int_{U^{2}} f(\zeta)\left(1-|\theta|^{2}\right) \frac{\partial}{\partial \theta} g_{0}(\theta, \zeta) \bar{\theta}^{2}\left(U_{\gamma_{\zeta}^{-1}} \varphi\right)(\theta) \overline{\psi(\zeta)}\left(1-|\zeta|^{2}\right)^{-r / 2} d \mu_{r}(\zeta) d \mu_{r}(\theta) \\
& -\pi^{-1} \int_{U^{2}} f(\zeta)\left(1-|\theta|^{2}\right) g_{0}(\theta, \zeta) \bar{\theta}^{2} \frac{\partial}{\partial \theta}\left(U_{\gamma_{\zeta}^{-1}} \varphi\right)(\theta) \overline{\psi(\zeta)}\left(1-|\zeta|^{2}\right)^{-r / 2} d \mu_{r}(\zeta) d \mu_{r}(\theta) .
\end{aligned}
$$

The first term on the right-hand side can be estimated by

$$
\begin{aligned}
& 2 \pi^{-1} \sup _{\zeta}|f(\zeta)| \sup _{\zeta, \theta}\left|\left(1-|\zeta|^{2}\right)^{-1}(1-|\theta|)^{8} \frac{\partial}{\partial \theta} g_{0}(\theta, \zeta)\right| \\
& \quad\left\{\int_{U}|\theta|^{4}(1-|\theta|)^{-14} d \mu_{r}(\theta)\right\}^{1 / 2}\left\{\int_{U}\left(1-|\zeta|^{2}\right)^{2-r} d \mu_{r}(\zeta)\right\}^{1 / 2}\|\varphi\|\|\psi\| \\
& \leqq C r^{-3 / 2}\|\varphi\|\|\psi\|,
\end{aligned}
$$

where we have used that

$$
\int_{U}|\theta|^{4}(1-|\theta|)^{-14} d \mu_{r}(\theta) \leqq O(1) r^{-3},
$$

and

$$
\pi^{-1} \int_{U}\left(1-|\zeta|^{2}\right)^{2-r} d \mu_{r}(\zeta)=1
$$

To estimate the second term in (VI.24) we first use (VI.13) and integrate by parts with respect to $\zeta$. The result is

$$
\begin{aligned}
& \pi^{-1} \int_{U^{2}} \partial f(\zeta)\left(1-|\theta|^{2}\right) g_{0}(\theta, \zeta) \bar{\theta}^{2}\left(U_{\gamma_{\zeta}^{-1}} \varphi\right)(\theta) \overline{\psi(\zeta)}\left(1-|\zeta|^{2}\right)^{-r / 2+1}(1+\bar{\zeta} \theta)^{-1} d \mu_{r}(\zeta) d \mu_{r}(\theta) \\
& \quad+\pi^{-1} \int_{U^{2}} f(\zeta)\left(1-|\theta|^{2}\right) \frac{\partial}{\partial \zeta}\left\{\left(1-|\zeta|^{2}\right)^{-1} g_{0}(\theta, \zeta)\right\} \\
& \quad \cdot \bar{\theta}^{2}\left(U_{\gamma_{\zeta}^{-1}} \varphi\right)(\theta) \overline{\psi(\zeta)}\left(1-|\zeta|^{2}\right)^{-r / 2+2}(1+\bar{\zeta} \theta)^{-1} d \mu_{r}(\zeta) d \mu_{r}(\theta) .
\end{aligned}
$$

Using (VI.22) (with $s=1$ ) and properties (i)-(iii) above, we estimate each of these terms by

$$
\begin{aligned}
& C\|\varphi\|\|\psi\|\left\{\int_{U}|\theta|^{4}(1-|\theta|)^{-16} d \mu_{r}(\theta)\right\}^{1 / 2}\left\{\int_{U}\left(1-|\zeta|^{2}\right)^{-r+2} d \mu_{r}(\zeta)\right\}^{1 / 2} \\
& \leqq C r^{-3 / 2}\|\varphi\|\|\psi\| .
\end{aligned}
$$

This completes the proof for $j=0$.

Let $j=1$. Using (VI.23) and integrating by parts with respect to $\theta$ we find that

$$
\begin{aligned}
& \pi^{-1}(r-1) \int_{U^{2}} f(\zeta) g_{1}(\theta, \zeta) \theta \bar{\theta}^{2}\left(U_{\gamma_{\zeta}^{-1}} \varphi\right)(\theta) \overline{\psi(\zeta)}\left(1-|\zeta|^{2}\right)^{-r / 2} d \mu_{r}(\zeta) d \mu_{r}(\theta) \\
& =-\pi^{-1} \int_{U^{2}} f(\zeta)\left(1-|\theta|^{2}\right) \frac{\partial}{\partial \theta} g_{1}(\theta, \zeta)|\theta|^{2}\left(U_{\gamma_{\zeta}^{-1}} \varphi\right)(\theta) \overline{\psi(\zeta)}\left(1-|\zeta|^{2}\right)^{-r / 2} d \mu_{r}(\zeta) d \mu_{r}(\theta) \\
& \left.\quad-\pi^{-1} \int_{U^{2}} f(\zeta)\left(1-|\theta|^{2}\right) g_{1}(\theta, \zeta)|\theta|^{2} \frac{\partial}{\partial \theta}\left(U_{\gamma_{\zeta}^{-1}} \varphi\right) \theta\right) \overline{\psi(\zeta)}\left(1-|\zeta|^{2}\right)^{-r / 2} d \mu_{r}(\zeta) d \mu_{r}(\theta)
\end{aligned}
$$




$$
-\pi^{-1} \int_{U^{2}} f(\zeta)\left(1-|\theta|^{2}\right) g_{1}(\theta, \zeta) \bar{\theta}\left(U_{\gamma_{\zeta}^{-1}} \varphi\right)(\theta) \overline{\psi(\zeta)}\left(1-|\zeta|^{2}\right)^{-r / 2} d \mu_{r}(\zeta) d \mu_{r}(\theta) .
$$

The first two terms on the right-hand side of this equation can be estimated in the same way as the terms in (VI.24) in the case of $j=0$. To estimate the third term we use (VI.23) (with $r$ replaced by $r+1$ ), integrate by parts with respect to $\theta$ (by doing so we produce the missing factor of $O\left(r^{-1}\right)$ ), and use the Schwarz inequality as in the analysis of $j=0$. The resulting bound is $\mathrm{Cr}^{-3 / 2}\|\varphi\|\|\psi\|$.

Let $j=2$. We use

$$
\theta\left(1-|\theta|^{2}\right)^{r-2}=\frac{1}{r-1} \frac{\partial}{\partial \bar{\theta}}\left(1-|\theta|^{2}\right)^{r-1},
$$

and integrate by parts with respect to $\theta$ to obtain

$$
\begin{aligned}
& \pi^{-1}(r-1) \int_{U^{2}} f(\zeta) g_{2}(\theta, \zeta) \theta^{2} \bar{\theta}\left(U_{\gamma_{\zeta}^{-1}} \varphi\right)(\theta) \overline{\psi(\zeta)}\left(1-|\zeta|^{2}\right)^{-r / 2} d \mu_{r}(\zeta) d \mu_{r}(\theta) \\
& =-\pi^{-1} \int_{U^{2}} f(\zeta)\left(1-|\theta|^{2}\right) \frac{\partial}{\partial \bar{\theta}} g_{2}(\theta, \zeta)|\theta|^{2}\left(U_{\gamma_{\zeta}^{-1}} \varphi\right)(\theta) \overline{\psi(\zeta)}\left(1-|\zeta|^{2}\right)^{-r / 2} d \mu_{r}(\zeta) d \mu_{r}(\theta) \\
& \quad-\pi^{-1} \int_{U^{2}} f(\zeta)\left(1-|\theta|^{2}\right) g_{2}(\theta, \zeta) \theta\left(U_{\gamma_{\zeta}^{-1}} \varphi\right)(\theta) \overline{\psi(\zeta)}\left(1-|\zeta|^{2}\right)^{-r / 2} d \mu_{r}(\zeta) d \mu_{r}(\theta) .
\end{aligned}
$$

Both terms on the right-hand side of this equation have a familiar structure and, by the methods explained above, they can be estimated by $\mathrm{Cr}^{-3 / 2}\|\varphi\|\|\psi\|$.

Finally, let $j=3$. Using (VI.27) and integrating by parts with respect to $\theta$ we obtain

$$
\begin{aligned}
& \pi^{-1}(r-1) \int_{U^{2}} f(\zeta) g_{3}(\theta, \zeta) \theta^{3}\left(U_{\gamma_{\zeta}^{-1}} \varphi\right)(\theta) \overline{\psi(\zeta)}\left(1-|\zeta|^{2}\right)^{-r / 2} d \mu_{r}(\zeta) d \mu_{r}(\theta) \\
& \quad=-\pi^{-1} \int_{U^{2}} f(\zeta)\left(1-|\theta|^{2}\right) \frac{\partial}{\partial \bar{\theta}} g_{3}(\theta, \zeta) \theta^{2}\left(U_{\gamma_{\zeta}^{-1}} \varphi\right)(\theta) \overline{\psi(\zeta)}\left(1-|\zeta|^{2}\right)^{-r / 2} d \mu_{r}(\zeta) d \mu_{r}(\theta) .
\end{aligned}
$$

Again, as in the case of $j=0$, this can be estimated by $C r^{-3 / 2}\|\varphi\|\|\psi\|$.

In conclusion, we notice that formula (VI.9) provides a systematic basis for generating an asymptotic expansion of $T_{f} T_{g}$ in powers of $r^{-1}$. The methods we have used to estimate the second order remainder in this expansion can presumably be used to estimate the remainder of arbitrary order.

Acknowledgements. We would like to express our gratitude to Arthur Jaffe, Palle Jorgensen, Marc Rieffel and especially Raul Curto for very helpful discussions.

\section{References}

1. Axler, S., Conway, J. B., McDonald, G.: Toeplitz operators on Bergman spaces. Can. J. Math. 34, 466-483 (1982)

2. Bayen, R., Flato, M., Fronsdal, C., Lichnerowicz, A., Sternheimer, D.: Deformation theory and quantization. Ann. Phys. 100, 61-151 (1978)

3. Bellisard, J.: K-theory of $\mathbb{C}^{*}$-algebras in solid state physics. Lecture Notes in Physics vol 257, pp. 99-156. Berlin, Heidelberg, New York: Springer 1986

4. Berezin, F. A.: General concept of quantization. Commun. Math. Phys. 40, 153-174 (1975) 
5. Brown, L. G., Douglas, R. G., Fillmore, P. A.: Extensions of $\mathbb{C}^{*}$-algebras and K-homology. Ann. Math. 105, 265-324 (1977)

6. Coburn, L. A.: Singular integral operators and Toeplitz operators on odd spheres. Indiana Univ. Math. J. 23, 433-439 (1973)

7. Connes, A.: A survey of foliations and operator algebras. Proc. Symp. Pure Math. 38, 521$628(1982)$

8. Connes, A.: Non-commutative differential geometry. Publ. Math. IHES 62, 94-144 (1986)

9. Connes, A., Moscovici, H.: Cyclic cohomology, the Novikov conjecture and hyperbolic groups. Topology 29, 345-388 (1990)

10. Douglas, R. G.: Banach algebra techniques in operator theory. New York, San Francisco, London: Academic Press 1972

11. Klimek, S., Lesniewski, A.: to appear

12. Klimek, S., Lesniewski, A.: to appear

13. Moore, G.: Geometry of the string equations. Commun. Math. Phys. 133, 261-304 (1990)

14. Moreno, C., Ortega-Navarro, P.: Deformations of the algebra of functions on Hermitian symmetric spaces resulting from quantization. Ann. Inst. H. Poincaré 38, 215-241 (1983)

15. Petersson, H.: Zur Analytischen Theorie der Grenzkreisgruppen, Teil I. Math. Z. 115, 23-67 (1938)

16. Podles, P.: Quantum spheres. Lett. Math. Phys. 14, 193-202 (1987)

17. Rieffel, M.: Deformation quantization of Heisenberg manifolds. Commun. Math. Phys. 122, 531-562 (1989)

18. Rieffel, M.: Non-commutative Tori - a case study of non-commutative differentiable manifolds. Contemp. Math. 105, 191-211 (1990)

19. Rieffel, M.: Deformation quantization and operator algebras. Proc. Symp. Pure Math. 51, 411-423 (1990)

20. Sheu, A. J.-L., Lu, J.-H., Weinstein, A.: Quantization of the Poisson $S U(2)$ and its Poisson Homogeneous space - the 2-Sphere. Commun. Math. Phys. 135, 217-232 (1991)

21. Unterberger, A. J.: A quantization of the Cartan domain $B D I(q=2)$ and operators on the light cone. J. Funct. Anal. 72, 279-319 (1987)

22. Woronowicz, S. L.: Twisted $S U$ (2) group: an example of non-commutative differential calculus. Publ. RIMS 23, 117-181 (1987)

Communicated by A. Jaffe 